\title{
Lyapunov Exponents of a Micro Chaotic Mixer
}

\author{
Yi-Kuen Lee \\ Department of Mechanical Engineering, Hong Kong University of Science and Technology, \\ Clear Water Bay, Kowloon, Hong Kong SAR, China, Email: meyklee@ust.hk
}

\begin{abstract}
In this paper, we study the Lyapunov exponents of a micro chaotic mixer to understand the mixing effectiveness under different operating parameters. A simple kinematic micro mixer model is used for the simulated flow visualization and then for the computation of Lyapunov exponents using two different numerical methods. These two methods generate consistent result to corroborate the existence of positive Lyapunov exponent which is the key signature of a chaotic system.
\end{abstract}

Keywords: micro mixer, chaotic mixing, microfluidics, MEMS, Lyapunov exponent, chaos

\section{Introduction}

Micromixers have been one of major research topics in microfluidics [1,2] because of many applications such as micro total analysis system ( $\mu$-TAS), high-speed chemical reaction study, fast pathogen detection system to counteract bioterrorism. The ultimate goal of micromixers is to efficiently mix a variety of bio-reactants such as bacteria cells, large DNA molecules, restriction enzymes, and proteins in portable integrated Microsystems with minimum power. However, mixing on the micro scale is usually difficult to achieve by generating turbulence because viscous effects dominate in the flow behavior. It has been reported that the inlet pressure for a microchannel of $102 \mu \mathrm{m}$ diameter is $1,800 \mathrm{psi}$ to achieve turbulent water flow $(\operatorname{Re}=19,000)$. This condition can cause safety problems in fabrication and testing [3].

On the other hand, the laminar nature of microchannel flows requires novel approaches to enhance mixing. Chaotic mixing is a promising solution since chaotic advection can occur at any Reynolds number. It is the degree of spatial-temporal complexity of the flow that determines whether the advected particle paths are chaotic, and not the force balance in the momentum equation. In recent years, there are many micromachined chaotic mixers proposed for integrated microfluidic systems [1-4]. However, there exists few research on how to quantitatively characterize the variety of micro mixers. In this paper, we will demonstrate the Lyanpunov exponents to be a possible mixing index for micro mixers since it is related to fluid stretching rate - an essential physical quantity for fluid mixing. Two different numerical methods will be used to calculate the Lyapunov exponents of a micro chaotic mixer model using periodic hydrodynamic forcing.

\section{Kinematic Model of Micro Mixer}

Finding the velocity field in a mixing flow from either experiments or theory is the beginning to understand the mixing structure in a given flow. We will first demonstrate poor mixing in 2-D Poiseuille flow by using the analytical velocity field and then propose a simple kinematic model for our micro mixer based on the Poiseuille flow.

For 2-D Poiseuille flow without any 
perturbation, the fluid stretching rate decreases as $1 / t$. This results in poor mixing. The line stretch with original orientation vector $m=\left(M_{1}\right.$, $M_{2}$ ) can be found from the deformation gradient tensor $d_{i j}$ of the flow with maximum velocity $V_{0}$. Therefore, the specific stretching rate $R_{\mathrm{s}}$ can be calculated by normalizing with respect to $\left(d_{i j} d_{i j}\right)^{1 / 2}=\sqrt{2} V_{o} X_{2}$ :

$$
R_{s}=\frac{1}{\left(d_{i j} d_{i j}\right)^{1 / 2}} \frac{D \ln \lambda}{D t}=\frac{2 \sqrt{2} M_{2}^{2} X_{2} V_{o} t-\sqrt{2} M_{1} M_{2}}{M_{1}^{2}+M_{2}^{2}-4 M_{1} M_{2} V_{o} t X_{2}+4\left(M_{2} V_{o} t X_{2}\right)^{2}}
$$

where $X_{1}, \quad X_{2}$ are the material coordinates in the streamwise and spanwise directions respectively [5]. Initially at $t=0, R_{s}$ is simply

$$
R_{s}=\frac{-\sqrt{2} M_{1} M_{2}}{M_{1}^{2}+M_{2}^{2}} .
$$

The maximum $R_{s}=1 / \sqrt{2}$ we found is consistent with the previous work by Khakhar et al. [6]. In order to improve mixing in the aforementioned channel flow, the reorientation of the fluid elements in the flow is important. Usually, stretching and folding by periodic temporal or spatial perturbations on the flow can achieve this goal, several side channels are added, perpendicular to the main channel flow, and act as control ports which provide sinusoidal pressure fluctuation to induce stretching and folding.

A micro chaotic mixer with simple designs were fabricated by micromachining technology including photolithography, deep silicon etching technique, etc [7]. A syringe pump drove the main stream in a micro channel. Pressure perturbation was applied transversally by means of three adjacent channels, connected to controlled pressure reservoirs. Without perturbation, an interface separating two miscible fluids (one fluid is labeled with fluorescent dye) remains sharp in the whole fluidic system. As properly designed pressure perturbation are found and activated, a sequence of folding and stretching takes place, revealing the presence of a special flow pattern as shown in Fig. 1(a).

In order to understand the mixing mechanism of our fabricated micormixer, we assume the main channel flows from left to right, and there is one side channel at the origin of the coordinates with channel width. The velocity profiles, both in the main and the side channel far from the intersection, are assumed to be parabolic (Poiseuille flow), with a constant and time dependent amplitude respectively. At the intersection between the control ports and the main channel, the velocity components from both channels are added together. The velocity field can be divided into three regions, I, II and III. Although this is a crude representation of the flow in this region, however, we believe this assumption does not affect the qualitative results as pointed out recently by Volpert et al. [8].

With this model, we first examine the interface pattern of two fluid blobs for different frequencies (so period $T=2 \pi$ ) by tracking 10,000 particles. As shown in Fig. 1(b), a periodic stretching and folding process can be observed in the simulated flow field, consistent with our experimental results in Fig.1(a). Obviously, to get truly chaotic regimes, the intersection region must be replicated indefinitely. In the microfluidic chip, it is very easy to make an array of side channels with periodic perturbation. To visualize this perturbation easily, we create a periodic boundary condition for our micromixer where the fluid particles moving out of one unit of the micromixer from the right boundary will move back to the left boundary. In this manner, an elegant chaotic-like fluid pattern is generated as shown in Fig. 2.

\section{Stretching Rate \& Lyapunov Exponent}

In order to quantatively characterize the mixing effectiveness using the mixer model discussed in the previous section, we use the mathematical identity which relates the specific stretching rate $D \ln \lambda / D t$ to the Lyapunov exponents (LE) of the system, $\sigma[5]$ :

$$
\sigma\left(\mathbf{X}, \mathbf{M}_{\mathbf{i}}\right)=\lim _{t \rightarrow \infty}\left\{\frac{1}{t} \int_{0}^{t} \frac{D \ln \lambda}{D t}\right\} d t^{\prime}=\lim _{t \rightarrow \infty}\left(\frac{1}{t} \ln \lambda\right) .
$$

Obviously, the LE is the long time average of the stretching rate. In this manner, the 
mixing effectiveness can be easily examined by looking at the LE, especially the largest one. On the other hand, from dynamic system theory, LE describes the mean exponential increase or decrease of small perturbations on the trajectories of a dynamic system. When the largest LE of a dynamic system is positive, the system is chaotic.

There are many other approaches to identify chaos quantitatively, such as fractal dimension, entropy. However, estimation of the LE from the numerical model is a well-known method for chaotic system identification. In recent years, many different methods to compute LE from time series have been developed [9]. It can be generally categorized into two approaches: Jacobian-based methods and direct methods. We have implemented these two different methods in computer programs ( $\mathrm{C}$ and Matlab) to calculate the LE of the micromixer model. In order to verify these programs, we calculate the LE of several classical chaotic systems (Lorenz attractors, Rössler attractor, and Ueda attractor) and compare with the previous papers as listed in Table 1. The good agreement of this calculation leads us to use the developed programs for the micromixer model with confidence. The convergence of the maximum micromixer LE for three different frequencies as $t$ approach infinity is confirmed as shown in Fig. 3.

In addition, from the Jacobian-based method, we found all three Lyapunov exponents: zero, positve and negative of the value found by the direct method. From these two different methods, the maximum LE curves as the function of normalized driving frequency are self-consistent as shown in Fig. 4.

\section{Conclusion}

The Lyapunov exponents of the proposed micromixer model have been successfully calculated using two different methods which were verified by several classical chaotic systems. These results show the nonlinear micromixer is a true chaotic system because of the existence of positive Lyapunov exponent. Since the definition of the Lyapunov exponent is quite general, it can be used to quantitatively characterize different micromixers in a consistent way.

\section{Acknowledgments}

The author would like to thank Prof Tong Xi $\mathrm{Yu}$ and Shilun Ruan at HKUST, Prof Chih-Ming Ho, H. Chen and S. Huang at UCLA and Prof Patrick Tabeling at CNRS, Paris for their assistance.

\section{References}

[1] Stroock, A.D. et al., Chaotic Mixer for Microchannels, Science, 295 (2002) 647 651.

[2] Liu, R. H., et al., Passive mixing in a three-dimensional serpentine microchannel, $J$. MEMS, 9(2) (2000)190-7.

[3] Yu, D. et al, An Experimental and Theoretical Investigation of Fluid Flow and Heat Transfer in Microtubes, ASME/JSME Thermal Engineering Joint Conf, (1995) 523-530.

[4] Evans, J., et al., Planar Laminar Mixer, Proceeding of the IEEE 10th Annual Workshop of MEMS (MEMS '97), Nagoya, Japan, Jan, (1997) 96-101.

[5] Ottino, J.M., The Kinematics of Mixing: Stretching, Chaos, and Transport, Cambridge University Press, New York, 1989.

[6] Khakhar, D.V, Rising, H., and Ottino, J.M., Analysis of Chaotic Mixing in Two Model Systems, J. Fluid Mechanics, 172(1986) 419-451.

[7] Lee, Y.K., Tabeling, P., Shih, C. and Ho, C.M., Characterization of a MEMS Fabricated Mixing Device, Proceedings of MEMS, ASME IMECE, Orlando, Florida, Nov., (2000) 419-425.

[8] Volpert, M., et al., An Actively Controlled Micromixer, Proceeding of MEMS, ASME IMECE, Nashville, Tennessee, Nov., (1999) 483-487.

[9] Suyken, J. A. K. and Vandewalle, J., ed., Nonlinear Modeling: advanced black-box techniques, Kluwer, Netherlands, 1998.

[10] Wolf, A, et al., Determining Lyapunov Exponents from a Time Series, Physica D, 16D (3) (1985) 285-317. 


\begin{tabular}{|l|l|l|l|}
\hline & Parameters & Previous papers & Calculated \\
\hline Lorenz attractor & $\sigma=10, r=28, b=8 / 3$ & $0.906[9]$ & 0.904 \\
\hline Lorenz attractor & $\sigma=16, r=45.92, b=4$ & $1.50[10]$ & 1.504 \\
\hline Rössler attractor & $a=b=0.2, c=5.7$ & 0.0714 & 0.0711 \\
\hline
\end{tabular}

Table 1: Verification of the programs to calculate the Lyapunov exponents.

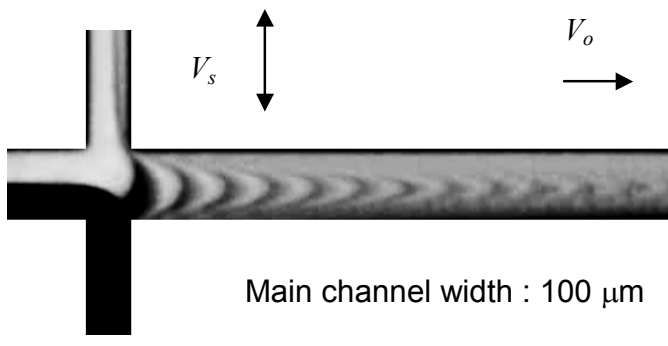

(a)

(b)

Fig. 1: (a) Micrograph shows the periodic hydrodynamic perturbation to enhance mixing of two fluids (black and white) in the microfabricated micromixer. (b) the kinematic simulation of two fluid blobs mixing by tracking 24,000 fluid particles.

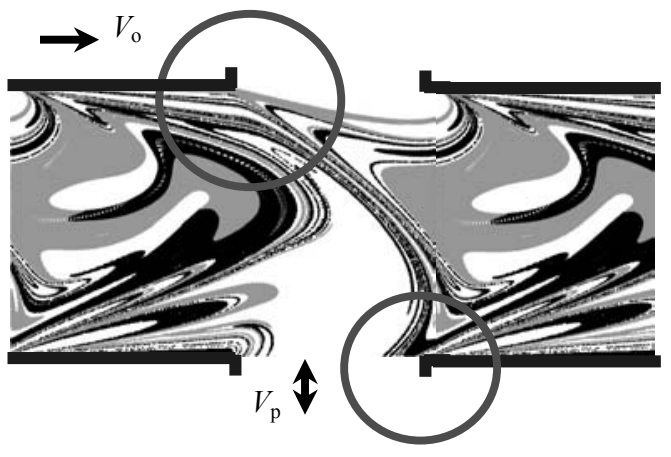

Fig. 2: Numerical computations show the role of the corners (red circles) as saddle points after 3 periods.

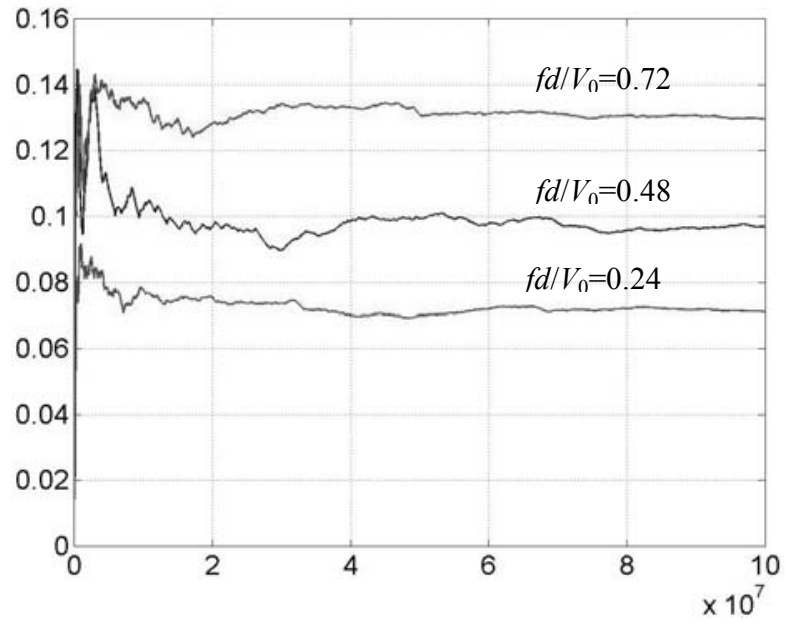

Fig. 3: Lyapunov exponent convergence plot for the micromixer for different $f d / V_{0}$ using the direct method.

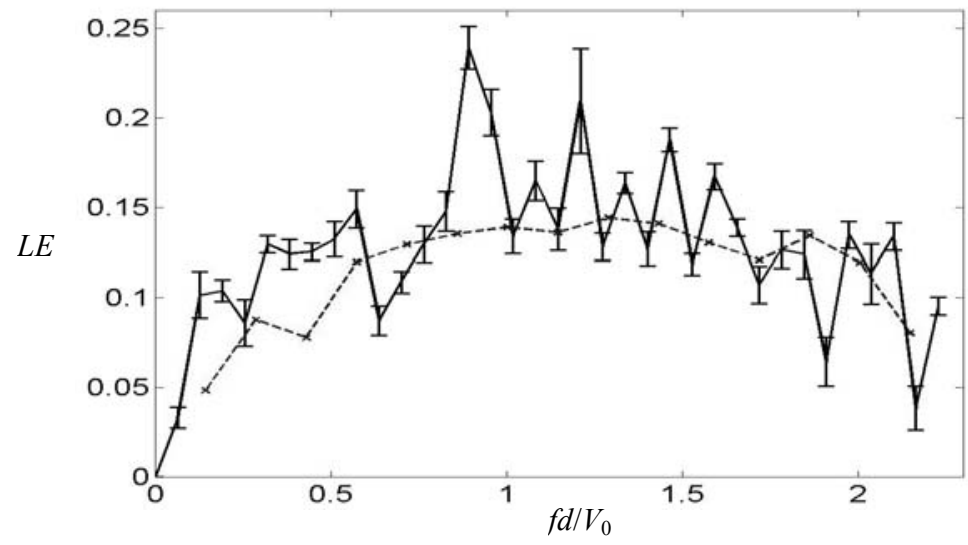

Fig. 4: Comparision of the Lyapunov exponent as a function of $\mathrm{fd} / \mathrm{v}$ with two methods: Direct method (dotted), Jacobian-based method (solid line with error bar). 